\title{
Wenn Frauen mehr als ihre Männer verdienen
}

\author{
Roberta Maria Koch
}

\section{Relevanz}

Die Frauen haben aufgeholt. Die steigenden Löhne der Frauen und ihre zunehmende Erwerbstätigkeit zeigen ihre wachsende Bedeutung in Wirtschaft und Gesellschaft. Der Trend setzt sich in den Familien fort. Die Familien, in denen die Frauen sich im Beitrag zum Familieneinkommen den Männern annähern, werden immer häufiger. Sobald jedoch die Frauen mehr als ihre Männer verdienen, nimmt der Anteil dieser Haushalte in der gesamten Bevölkerung schlagartig ab. Auch die Scheidungsraten nehmen in dieser Konstellation zu. Hoch qualifizierte Frauen machen bisweilen kostspielige Kompromisse, um Konflikten auszuweichen. Es scheint schwierig, mit traditionellen Rollenbildern zu brechen. Diesen Prozess zu beschleunigen, würde nicht nur der Qualität des Familienlebens nützen, sondern auch Wirtschaft und Gesellschaft zugutekommen.

\section{Quelle}

Marianne Bertrand, Emir Kamenica und Jessica Pan (2015), Gender Identity and Relative Income Within Households, Quarterly Journal of Economics 130(2), 571-614.

Frauen verdienen im Durchschnitt deutlich weniger als Männer. Dies trifft nicht nur auf die Schweiz zu, sondern zeigt sich in praktisch allen Ländern. Eine mögliche Erklärung für diese Ungleichheit sind soziale Normen hinsichtlich der

R. M. Koch $(\bowtie)$

Universität St.Gallen, St.Gallen, Schweiz

E-Mail: robertamaria.koch@student.unisg.ch 
Geschlechterrollen. Diese teilen Frauen wie Männern bestimmte Verhaltensweisen, Eigenschaften und Aufgaben zu. Der Mann geht zur Arbeit, die Frau sorgt für Kinder und Haushalt. Diese klassische Rollenverteilung ist auch in Zeiten der MeToo-Debatte und weltweiten Frauenmärschen weitverbreitet. Doch was passiert, wenn Frauen beruflich erfolgreicher sind als ihre Ehemänner und, entgegen ihrem traditionellen Rollenbild, zur Hauptverdienerin der Familie aufsteigen?

Obwohl Frauen nach wie vor für die gleiche Arbeit oft weniger verdienen als ihre männlichen Kollegen, ist die Lohndifferenz während der letzten Jahrzehnte gesunken. Im selben Zeitfenster nahm jedoch auch die Heiratsquote deutlich ab. Motiviert durch diese Entwicklung gingen Marianne Bertrand, Emir Kamenica und Jessica Pan der Frage nach, welche Auswirkungen es haben kann, wenn die Frau die Hauptverdienerin eines Haushalts ist. Zur Beantwortung dieser Frage verwendeten die drei Ökonominnen und Ökonomen der University of Chicago Daten aus den USA in den Jahren 1970-2000 und 2008-2011.

Abb. 1 illustriert die Ausganslage: Sie zeigt die Verteilung der Haushalte, ansteigend nach dem Einkommensanteil der Frauen in der Familie, zwischen 1990 und 2011. Berücksichtigt wurden ausschliesslich Haushalte, in denen beide Ehepartner berufstätig sind. Die horizontale Achse reiht die Haushalte nach dem Anteil, welchen die Ehefrau zum gesamten Haushaltseinkommen beisteuert. Die Punkte zeigen den Anteil von Ehepaaren, welche den jeweiligen Einkommensanteil der Frau aufweisen. Zum Beispiel sind es knapp $10 \%$ der Haushalte, in denen die Frau $40 \%$ des Familieneinkommens verdient.

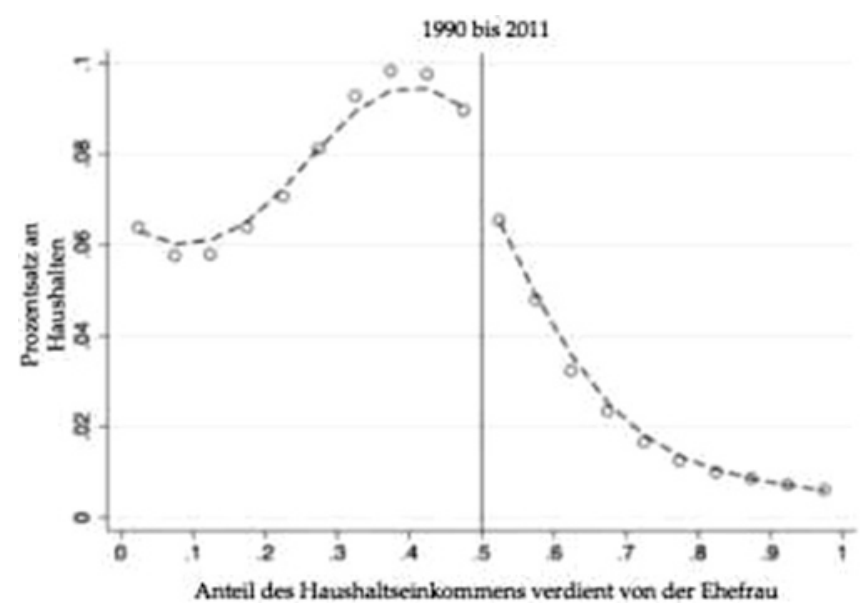

Abb. 1 Verteilung des relativen Haushaltseinkommens. (Quelle: Bertrand u. a. 2015) 
Die vertikale Linie hebt den Anteil der Haushalte hervor, bei welchem beide Partner gleich viel verdienen und somit jeweils die Hälfte zum Haushaltseinkommen beitragen. Deutlich erkennbar ist ein Bruch in der Verteilung, sobald die Frauen die Schwelle von $50 \%$ des Familieneinkommens übertreffen: Der Anteil der Haushalte sinkt schlagartig, sobald die Ehefrau mehr verdient als der Mann. Die Daten weisen beim Überschreiten dieser Schwelle einen Rückgang von 13.6\% auf. Dieser ist in allen untersuchten Jahren sichtbar, doch das Ausmass des Rückgangs hat sich in den letzten Jahrzehnten verringert. So belief sich dieser Sprung in den 80er-Jahren noch auf über ein Viertel. Im Zeitraum von 2008 bis 2011 fiel der Anteil der Haushalte beim Überschreiten dieses Verdienstanteils nur noch um rund $10 \%$. Immer mehr Frauen werden zur Hauptverdienerin der Familie.

Der Anteil der Haushalte steigt, in denen die Frauen zu den Männern aufholen und einen wachsenden Anteil des Familieneinkommens beisteuern. Sobald jedoch die Frau besser verdient als ihr Ehemann, geht der Anteil dieser Haushalte stark zurück. Über die Jahrzehnte hinweg hat sich das Ausmass des Rückgangs jedoch verringert. Immer mehr Frauen werden zur Hauptverdienerin der Familie.

Der Bruch in der Verteilung besteht auch bei Paaren ohne Kinder, ist aber bei Familien mit Kindern stärker ausgeprägt. Auch die Dauer der Ehe hat einen Einfluss darauf, um wie viele Prozentpunkte der Anteil der Haushalte abnimmt, wenn die Ehefrau mehr verdient als ihr Mann. Je frischer die Eheschliessung, umso schwächer fällt der Sprung aus. Bei Paaren, welche nicht länger als ein Jahr verheiratet sind, beträgt der Rückgang $8.4 \%$. Paare, welche seit 2 bis 5 Jahren verheiratet sind, weisen einen Rückgang von $10.1 \%$ auf. Unter allen Paaren, die zwischen 6 und 10 Jahren verheiratet sind, geht der Anteil der Paare um ganze $12.9 \%$ auf, sobald die Frau mehr als die Hälfte zum Familieneinkommen beisteuert.

In den letzten Jahrzehnten stiegen die Löhne der Frauen stetig an. Der Gehaltsunterschied zu den Männern ging zurück. Gleichzeitig nahm die Heiratsquote ab. Die Studienautoren vermuten einen Zusammenhang zwischen diesen beiden Entwicklungen. Im Beobachtungszeitraum stieg die Wahrscheinlichkeit, dass eine zufällig ausgewählte Frau mehr verdient als ein zufällig ausgewählter Mann, von 17-20 \% auf rund ein Drittel im Jahr 2010. Die Wahrscheinlichkeit ist wesentlich grösser geworden, dass die Einkommensverteilung innerhalb eines Haushalts dem traditionellen Rollenbild widerspricht, wonach der Ehemann Hauptverdiener ist. Die Schätzungen der Wissenschaftler zeigen, dass dieser relative Lohnanstieg der Frauen rund $29 \%$ des Rückgangs der Heiratsrate von 1980 bis 2010 erklären kann.

Der Lohnanstieg der Frauen im Vergleich zu Männern kann rund 29 Prozent des Rückgangs der Heiratsrate im Zeitraum von 1980 bis 2010 erklären. 
Wie reagieren Ehefrauen darauf, dass ihr relativer Lohnzuwachs die traditionelle Rollenverteilung infrage stellt? Die Frauenerwerbsquote in den USA, welche 1970 nur bei $43 \%$ lag, hat deutlich zugenommen, jedoch stagniert sie seit Mitte der 1990er Jahre bei knapp 75 \%. Die Studienautoren argumentieren, dass Geschlechterrollen zumindest teilweise für diese Stagnation verantwortlich sind. Manche Frauen, deren potenzielles Einkommen jenes ihres Mannes übertrifft, entschliessen sich sogar, ihre Erwerbstätigkeit zu verringern oder ganz aufzugeben. Steigt die Wahrscheinlichkeit, dass die Frau mehr verdient als ihr Ehemann, um zehn Prozentpunkte, dann sinkt die Wahrscheinlichkeit, dass sie einer Erwerbstätigkeit nachgeht, um rund 1.4 Prozentpunkte bzw. um zwei Prozent. Insgesamt schliessen Bertrand und ihre Koautoren daraus, dass verheiratete Frauen teilweise bewusst nicht arbeiten, um nicht zur Hauptverdienerin des Haushalts aufzusteigen. Dieses Verhalten ist besonders bei Paaren mit niedriger Bildung zu beobachten. Einkommensverzicht oder gar der Ausstieg der Frau aus dem Arbeitsmarkt sind äusserst kostspielige Verhaltensweisen, um die traditionelle Rollenverteilung zu bewahren.

Manche Frauen ziehen sich bewusst aus der Erwerbstätigkeit zurück, um nicht die Haupternährerin der Familie zu sein. Eine um zehn Prozentpunkte höhere Wahrscheinlichkeit, dass die Frau mehr verdient als ihr Mann, reduziert die Wahrscheinlichkeit ihrer Erwerbstätigkeit um rund zwei Prozent.

Dennoch kommt es immer öfter vor, dass Frauen mehr verdienen als ihre Ehemänner. Im Jahr 2010 traf dies auf $27 \%$ der Ehepaare in den USA (1865 Jahre) zu. In diesem Fall könnten Frauen versucht sein, die „Verletzung“ der traditionellen Geschlechterrolle dadurch zu kompensieren, dass sie mehr Hausarbeit leisten als ihre Ehemänner, selbst wenn letztere deutlich weniger verdienen. Die empirischen Ergebnisse zeigen tatsächlich, dass das Geschlechtergefälle bei der Hausarbeit stärker zu Ungunsten der Frauen ausfällt, wenn sie ihre Männer im Verdienst übertreffen.

Dies könnte nach Bertrand, Kamenica und Pan einer der Gründe sein, weshalb Paare mit der Frau als Hauptverdienerin öfter Eheprobleme aufweisen bzw. sich öfter scheiden lassen. Wenn die Frau neben ihrer Erwerbstätigkeit auch noch einen Grossteil der Hausarbeit auf sich nimmt, ist sie stärker belastet. Die Qualität der Ehe leidet in dieser Situation. Die Schätzungen ergeben: Wenn die Frau vor zwei Jahren mehr verdient hat als der Mann, liegt die Wahrscheinlichkeit einer Scheidung um rund ein Viertel höher als in den klassischen Fällen mit den Männern als Hauptverdiener.

Insgesamt zeigt die Studie, welchen nicht zu unterschätzenden Einfluss soziale Geschlechternormen auf die Verteilung des Einkommens innerhalb eines 
Haushalts sowie auf die Erwerbstätigkeit von Frauen und auf die Qualität des Zusammenlebens haben können. Die Löhne für Frauen sind in den letzten Jahrzehnten deutlich angestiegen. Geschlechterrollen und Verhaltensweisen passen sich dagegen nur langsam an. Die Starrheit der sozialen Normen ist schwierig zu überwinden und bringt hohe Kosten sowohl für die Eheleute wie auch für die Gesellschaft als Ganzes mit sich.

Open Access Dieses Kapitel wird unter der Creative Commons Namensnennung 4.0 International Lizenz (http://creativecommons.org/licenses/by/4.0/deed.de) veröffentlicht, welche die Nutzung, Vervielfältigung, Bearbeitung, Verbreitung und Wiedergabe in jeglichem Medium und Format erlaubt, sofern Sie den/die ursprünglichen Autor(en) und die Quelle ordnungsgemäß nennen, einen Link zur Creative Commons Lizenz beifügen und angeben, ob Änderungen vorgenommen wurden.

Die in diesem Kapitel enthaltenen Bilder und sonstiges Drittmaterial unterliegen ebenfalls der genannten Creative Commons Lizenz, sofern sich aus der Abbildungslegende nichts anderes ergibt. Sofern das betreffende Material nicht unter der genannten Creative Commons Lizenz steht und die betreffende Handlung nicht nach gesetzlichen Vorschriften erlaubt ist, ist für die oben aufgeführten Weiterverwendungen des Materials die Einwilligung des jeweiligen Rechteinhabers einzuholen.

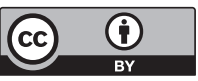

